\title{
Chernobyl's legacy to science
}

The consequences of the Chernobyl accident have given health physicists and geneticists a wealth of information about the effects of radiation exposure. Some is reassuring, some less so, but support for structured follow-up studies remains essential.

IT might be seen as churlish to look for a silver lining in a cloud as grey and threatening as that caused by the explosion of the Unit 4 reactor at Chernobyl, exactly ten years ago tomorrow. Whatever the precise figures involved turn out to be, the human suffering involved was immense. It includes that of the tens of firemen who died after fighting the fire that followed, of the many hundreds of children who have since developed thyroid cancers from ingesting radioactive iodine, and of the hundreds of thousands of people who suffered severe psychological stress as a result of having to leave their homes and communities.

Furthermore, as last weekend's G7 summit meeting in Moscow demonstrated, the danger is not yet over. Few are confident about the safety of nuclear reactors in the former Soviet Union, and many will sleep peacefully only when the 15 Chernobyl-style reactors - including those still operating at Chernobyl itself - have been taken out of service.

It would, however, be equally churlish to deny the extent to which medical, environmental and scientific studies of the consequences of the accident in the intervening ten years have helped researchers to refine their knowledge of the impact of radiation on living organisms. This knowledge has many important implications. To take but one example, the cost-effectiveness of the massive effort being undertaken in the United States to clean up the nuclear facilities of the Department of Defense depend critically on the social acceptability of radiation levels that will be left after the clean-up has been completed. If there is a threshold below which radiation has no long-term biological effect, will much be gained by achieving complete elimination? Conversely, if no threshold exists, can the costs of eliminating radiation risks entirely be justified by the likely medical benefits if these are, ultimately, insignificantly small?

In this context, the Chernobyl accident has at the least provided researchers with a unique scientific and medical experiment. Some of the lessons were immediate; one, for example, was the relative ineffectiveness of the bone-marrow transplantation used in a desperate attempt to save some of those who had received massive exposures after the accident. Such transplants can work in a hospital setting, where closely controlled radiation can ensure the complete elimination of an individual's own bone marrow before its replacement. But where an individual's exposure has been uncontrolled and uneven, residual bone marrow, we now know from experience, quickly sets up an immune response leading to rejection of the implant.

Other lessons have, at least so far, been relatively reassuring for health physicists. The high levels of thyroid cancer among children in the region, with more than 400 such cases reported from Belarus since 1990, as well as the relative absence of an increased level among other age groups, are broadly in line with previous expectations, based on calculations of the likely doses that would have been received by members of families in which advice to take iodine prophylaxis and not to eat home-grown vegetables and locally produced milk were ignored or given too late. Conversely, the apparent absence so far of any documented leukaemia among exposed population groups is also consistent with predicted dose-response relationships based on a relatively low exposure to caesium in the ground.
Some of the potential lessons remain to be addressed. One tantalizing prospect facing cancer epidemiologists is that of carrying out mortality and morbidity surveys of the roughly 600,000 individuals who acted as so-called 'liquidators' clearing up the Chernobyl area after the accident. Both the relative consistency of the exposure received by these individuals, who were removed from the area as soon as such doses exceeded 100 millisieverts, and their genetic proximity to large potential control populations in Western Europe promise data that could be as significant in analysing the impact of radiation exposure as that gained from the Japanese atomic bomb survivors - the main data on which modern radiation standards are set, both for those working in the nuclear industry and for environmental exposure.

Finally, as two papers published in this issue of Nature illustrate, genetic studies of both human and animal populations, using techniques far more advanced than those available at the time of the Japanese studies, promise new insights into the precise way in which radiation interacts with living organisms - and how such organisms respond (see pages 683-686, 707-708 and 665 ). Some of these data are potentially disturbing; an unusually high level of length changes in nuclear minisatellite loci among individuals living even a few hundred kilometres away from the accident, providing apparent evidence of germline alterations due to low levels of radiation, merits further study despite uncertainties in mutation rates and radiation exposure. Other results are intriguing; one question raised by evidence of unexpectedly high rates of substitution in protein-coding genes in voles living near the stricken reactor is why these animals appear to be surviving successfully under high radiation levels.

Furthermore, Chernobyl could still spring some surprises, both in Russia and elsewhere. Few Western scientists are prepared to accept at face value claims made earlier this month by Ukrainian health officials that 125,000 people have already died as a result of the accident. Scepticism has also greeted other suggestions made last week that the death rate may be much higher than Western scientists are predicting (see page 658). Without the clear presentation of the relevant clinical evidence - including detailed comparison to control populations - in peer-reviewed journals, many in the West will be reluctant to accept conclusions that contradict conventional wisdom. Yet it would be foolhardy to reject all such claims on this basis; certainly the more plausible justify continued monitoring.

Much of the damage caused by the Chernobyl accident including the severe dent in the safety reputation of the world's nuclear industry - is likely to be irreversible. Much of it has yet to emerge; one of the most upsetting facts facing those studying its long-term consequences is that the incidence of thyroid cancers among exposed children is likely to remain high for many years to come. And much remains to be learnt of the long-term physical and psychological consequences. Perhaps the most significant compensation that politicians can offer for the enormous human and environmental costs of the Chernobyl accident would be to make sufficient funds available to ensure - for example by a detailed long-term study of the Chernobyl 'liquidators' - that the unique opportunity for increasing our knowledge of the true dangers of radiation is not lost. 